\title{
Insights into the reinforcement role of peritubular dentine subjected to
}

\section{acid dissolution}

\author{
Jianan $\mathrm{Hu}^{1}$, Tan $\mathrm{Sui}^{2 *}$ \\ ${ }^{1}$ Sente Software Ltd., 40 Occam Road, Surrey Technology Centre, Guildford, Surrey, GU2 7YG, UK \\ ${ }^{2}$ Department of Mechanical Engineering Sciences, University of Surrey, Guildford, Surrey, GU2 7XH, UK \\ *Corresponding author: t.sui@surrey.ac.uk
}

\begin{abstract}
Human dentine is a mineralised dental tissue that consists of dentinal tubules surrounded by two distinct dentinal phases: peritubular dentine (PTD) and intertubular dentine (ITD). Dental caries, which manifests itself as a consequence of demineralisation, is one of the most common chronic diseases that affect the function of human teeth. Due to the difference in the packing density of crystallites, PTD and ITD exhibit different reaction rates to acid dissolution. The present study evaluates how the effective Young's modulus degrades and how the effective stress redistributes in demineralised human dentine as a result of incremental acid dissolution process. An analytical two-layer composite model is proposed and used for the effective Young's modulus calculation. 3D numerical representative volume elements (RVEs) with different variations in PTD fraction and dentinal tubule density are established to evaluate effective stress redistribution and examine the critical factors that can affect the mechanical performance. The models are then applied on an actual dentine bulk sample. The results reveal how PTD serves as a protection to ITD thus highlight the important role that PTD plays for the structural integrity of dentine. The obtained insights are crucial for advancing the understanding of a variety of natural and therapeutic effects from the mechanical perspective, e.g. the mechanical performance assessment of human dentine subject to complex dynamic processes of de- and remineralisation that can occur in human dental caries and dental treatments. It will ultimately inspire the biomimetic design towards strengthening the dentine and dentine-like materials.
\end{abstract}


Keywords: peritubular dentine (PTD); intertubular dentine (ITD); finite element method; dental acid dissolution.

\section{Introduction}

Human dentine is a natural mineralised material that is densely perforated with dentinal tubules with a variation in the diameter $(\sim 2-3.5 \mu \mathrm{m})[1,2]$. These tubules are oriented perpendicular close to the dentine-enamel junction (DEJ) and extend to the pulp through the entire dentine following an Sshaped path [3-5]. The tubules are known to be surrounded by two distinct dentinal phases: peritubular dentine (PTD) and intertubular dentine (ITD), where PTD possesses a tube-like structure. The principal difference between PTD and ITD is the nanoscale packing density of the Hydroxyapatite (HAp) crystallites, making PTD more brittle while ITD more ductile [6]. The entire dentine structure is similar to a composite where ITD serves as the matrix and PTD serves as reinforcement. Fig. 1(a) provides a snapshot of a freshly extracted human third molar dentine slice sample with no dental restorations, caries or damage. It was reconstructed from a sequence of back-scattered electron (BSE) images obtained by sequential focused-ion beam (FIB) millings [7]. The figure has clearly distinguished PTD and ITD from the variation of grey value (intensity) and demonstrated the irregular spatial distribution of each constituent.

The microstructure and properties of human dentine have been investigated for decades. Efforts have been devoted to achieving a detailed observation of the hierarchical features and elemental distribution of dentinal tubules and the surroundings (e.g. PTD and ITD) using advanced characterisation facilities. For example, X-ray tomography technique has been used to visualise the spatial distribution of nanocrystalline HAp around the tubules and to quantify the differences in mineralisation density between ITD and PTD [8, 9]. Synchrotron X-ray diffraction and spectroscopy techniques have been used to map the elemental distribution from HAp [10, 11]. Focused-ion beam 
(FIB), scanning electron microscopy (SEM), energy dispersive spectroscopy (EDS) and tapping mode Atomic Force Microscopy (AFM) have been systematically utilised to achieve 3D spatially resolved structural and compositional characterisation of the arrangement of mineral and organic phases within ITD and PTD [12].

In parallel, mechanical tests, analytical modelling and numerical simulations have been conducted to assess the mechanical performance of dentine in different scenarios. For instance, the shear strength and ultimate tensile strength of dentine have been revealed to be dependent on the orientation of tubules and collagen fibrils $[13,14]$. Balooch et al [15] have demonstrated the anisotropic feature of dentine using AFM-based indentation. Micromechanics models of the anisotropic elastic stiffness of dentine bulk can be found in [16-18] which have taken into account the hierarchical structure. Localised elastic property variation of human dentine has also received extensive attention [19]. Wang et al [6] have demonstrated the reinforcement effect of PTD in the elastic property by using a two-dimensional (2D) numerical model of dentine and manipulating the sizes of PTD and dentinal tubules. An [20] performed computational simulations of fracture behaviour of a 2D dentine structure and revealed that the toughness and degree of microcracking are dependent on tubule density and PTD thickness. All these efforts have been driven by the desire to correlate the versatile structural features with the remarkable mechanical properties of dentine and how the biomimetic design can be facilitated by an improved understanding of the role of microstructure.

Beyond the intact healthy dentine, a growing effort has been dedicated to the research of dental caries, which is one of the most common chronic diseases that undermines the mechanical function and structural integrity of human teeth [21]. A direct result of dental caries is the demineralisation process that would lead to microstructural changes and crystallography of the inorganic mineral phase. AFM has been used to study these microstructural changes in dentine at early stages of acid dissolution (e.g. using a pH 4.0 lactic acid gel) [22, 23]. PTD and ITD have been found to react differently to the acid reaction with different dissolution rates [22]. This is depicted in Fig. 1(b), where PTD depth 
changes were linear while the ITD surface initially moved at a similar rate and then reached a plateau as the demineralised collagen scaffold collapsed. The remaining depths of PTD and ITD of the human third molar dentine slice sample after each dissolution step in Fig. 1(a) has been summarised in Tab. 1. In addition, other findings include no apparent difference in the tubule centre-to-centre distance during the treatment [23]. These observations have been useful to the understanding of the carious process in dentine. However, little is known about how the mechanical properties degrade as a result of the acid dissolution [24].

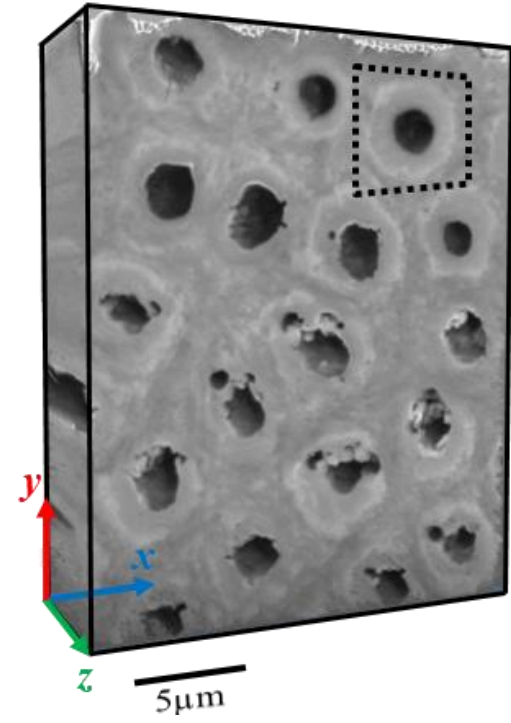

(a)

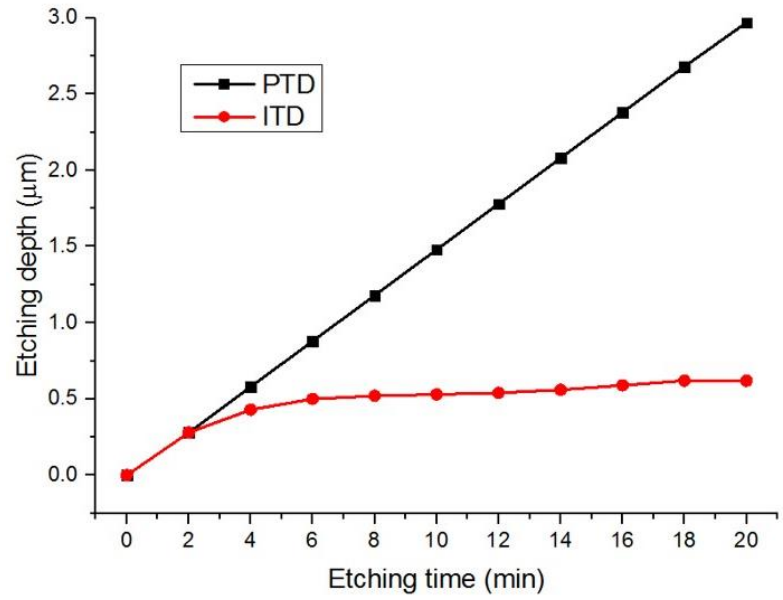

(b)

Fig. 1 (a) A 3D reconstruction of a representative dentine bulk structure composed of tubules, PTD and ITD, distinguishable by the contrast in greyscale intensity [7]. (b) Dissolution depth (in $\mu \mathrm{m}$ ) of PTD and ITD as a function of time (in min), extracted from [22].

Table 1 Depths of PTD and ITD after dissolution (original depth of the representative bulk of dentine is $7 \mu \mathrm{m}$ )

\begin{tabular}{|c|c|c|c|}
\hline Step No. & Time (min) & $\begin{array}{c}\text { Remaining depth } \\
\text { of PTD }(\boldsymbol{\mu m})\end{array}$ & $\begin{array}{c}\text { Remaining depth } \\
\text { of ITD }(\boldsymbol{\mu m})\end{array}$ \\
\hline $\mathbf{0}$ & 0 & 7 & 7 \\
\hline $\mathbf{1}$ & 2 & 6.72 & 6.72 \\
\hline $\mathbf{2}$ & 4 & 6.42 & 6.57 \\
\hline $\mathbf{3}$ & 6 & 6.12 & 6.5 \\
\hline $\mathbf{4}$ & 8 & 5.82 & 6.48 \\
\hline $\mathbf{5}$ & 10 & 5.52 & 6.47 \\
\hline $\mathbf{6}$ & 12 & 5.22 & 6.46 \\
\hline $\mathbf{7}$ & 14 & 4.92 & 6.44 \\
\hline $\mathbf{8}$ & 16 & 4.62 & 6.41 \\
\hline $\mathbf{9}$ & 18 & 4.32 & 6.38 \\
\hline $\mathbf{1 0}$ & 20 & 4.03 & 6.38 \\
\hline
\end{tabular}


In this paper, we first develop an analytical composite model of human dentine in relation to its unique PTD and ITD microstructure and then establish five 3D numerical representative volume elements (RVEs) in two groups with variations in either PTD fraction or dentinal tubule density to determine how the effective Young's modulus would degrade, to calculate mechanical response, in particular the effective stress redistribution subject to a mastication-like load, and to evaluate the critical factors that can influence the mechanical performance, as a consequence of acid dissolution. The dimension of each RVE is designed based on the information from the dentine bulk sample image characterised by SEM in Fig. 1(a). Both the effective Young's modulus degradation and effective stress redistribution of these RVEs are also compared with the results obtained using the actual dentine bulk sample geometry shown in Fig. 1(a). The objective of this paper is to determine the critical factors that influence the effective stress redistribution inside the demineralised dentine bulk and explore the role of PTD in the structural integrity of dentine from the mechanical perspective.

\section{Methodology}

\subsection{A composite model for demineralised dentine}

This section introduces a simple analytical composite model to describe the mechanical property, in particular the effective Young's modulus, of a representative bulk of dentine depicted in Fig. 1(a), subject to an acid dissolution along the tubule direction (Fig. 1b). A schematic model of dentine before acid dissolution is illustrated in Fig. 2(a), consisting of ITD, PTD and tubules in parallel. Initially PTD and ITD have identical depth (z-direction). After acid dissolution, as PTD dissolves more rapidly than ITD (Fig. 1b), the demineralised dentine can be regarded as a two-layer demineralised composite model (Fig. 2b). The top layer consists of ITD and tubules but with a different volume fraction from the intact composite model, whereas the bottom layer resembles the intact composite model but with a reduced depth. Here we focus on the effective Young's modulus of the dentine along the tubule direction (parallel to the acid dissolution direction, i.e. $z$-direction in Fig. 1), which is similar to the 
mastication direction. Therefore, the effective Young's modulus of each layer can be described by the well-known Voigt model [25], assuming the strains parallel to the tubule direction are identical for all the constituents.

$$
\begin{gathered}
E_{b}=f_{P T D} E_{P T D}+f_{I T D} E_{I T D}+f_{t u b} E_{t u b} \\
E_{t}=f_{I T D} E_{I T D}+f_{t u b}{ }^{\prime} E_{t u b}
\end{gathered}
$$

where the subscript ' $b$ ' denotes 'bottom layer' and the subscript ' $t$ ' denotes 'top layer', $f_{P T D}, f_{I T D}, f_{\text {tub }}$ and $E_{P T D}, E_{I T D}, E_{t u b}$ refer to the volume fraction and Young's modulus of PTD, ITD and tubules respectively $\left(f_{P T D}+f_{I T D}+f_{t u b}=1\right)$. Note $f_{t u b}$ ' has a different magnitude from that of the bottom layer because of the disappearance of PTD on top layer. Taking into account the dimensions shown in Fig. 2 and all the tubules and associated PTD:

$$
\begin{gathered}
f_{\text {tub }}=\sum_{i} \pi r_{i}^{2} / w l \quad f_{\text {PTD }}=\sum_{i} \pi\left(R_{i}^{2}-r_{i}^{2}\right) / w l \\
f_{\text {tub }}{ }^{\prime}=\sum_{i} \pi R_{i}^{2} / w l=f_{\text {tub }}+f_{\text {PTD }} \\
f_{\text {ITD }}=1-f_{\text {tub }}-f_{\text {PTD }}=1-\sum_{i} \pi R_{i}^{2} / w l
\end{gathered}
$$

For the entire demineralised composite model (Fig. 2b), as the top layer and bottom layer are packed along the loading direction, Reuss model [25] is more appropriate, assuming the effective stresses of the two layers are equal. Suppose at a stage of acid dissolution, the remaining depth of the top layer is $d_{t}$ and that of the bottom layer is $d_{b}$, according to the Reuss model, the effective Young's modulus ( $\left.E_{\text {eff }}\right)$ of the combined composite can be finally expressed as

$$
E_{\text {eff }}=\frac{E_{b} E_{t}}{\left(1-f_{b}\right) E_{b}+f_{b} E_{t}}
$$

where $f_{b}$ is the volume fraction of the bottom layer $f_{b} \approx d_{b} /\left(d_{b}+d_{t}\right)$. At the beginning, $d_{t}=0$ and $f_{b}=1$ so $E_{\text {eff }}=E_{b}$ as the original intact composite. Although Voigt (Eq. 1) and Reuss (Eq. 4) approximations are often considered to be upper and lower bounds, they allow to provide reasonable qualitative evolution of the effective Young's modulus of dentine during heterogeneous dissolution. 


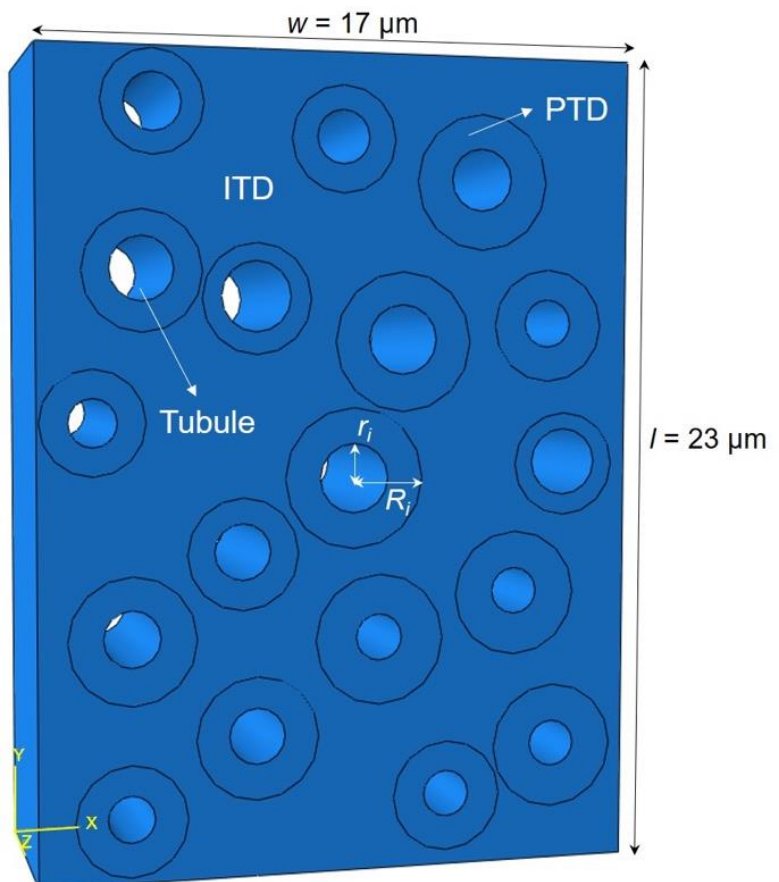

(a)

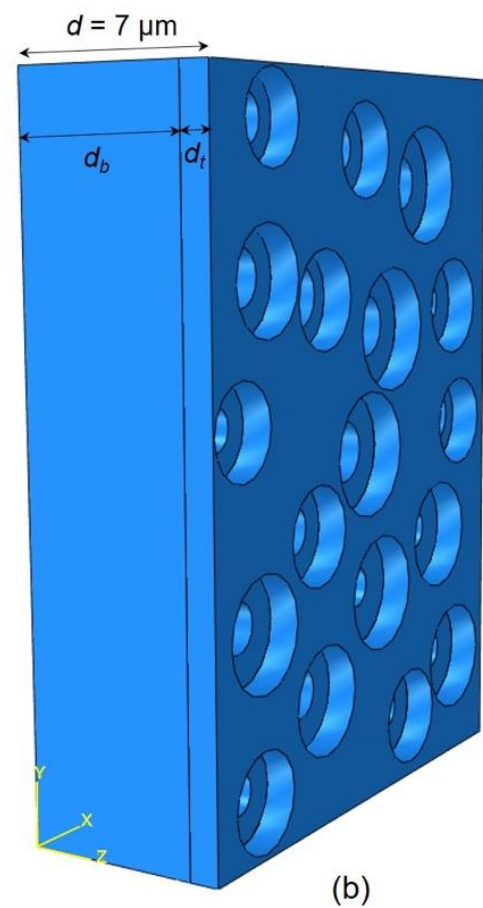

(b)

Fig. 2 A schematic geometry of a dentine bulk section (a) before and (b) during dissolution. All the dimensions are shown in the figure. $r_{i}$ is the radius of $i$ th tubule and the thickness of $i$ th PTD is $R_{i}-r_{i}$. During dissolution, the bulk can be regarded as a two-layer structure as a result of dissolution of PTD. The depth of top (bottom) layer is depicted as $d_{t}\left(d_{b}\right)$.

\subsection{RVEs of demineralised dentine}

The dentine bulk section consists of a number of tubules with various radii and PTD thicknesses. To illustrate and distinguish the critical factors that influence the mechanical performance for the dentine bulk as a consequence of acid dissolution, five equivalent 3D RVEs were established in finite element (FE) package ABAQUS ${ }^{\circledR} 6.14$ to simulate dentine bulks with uniform PTD and tubule sizes. A schematic and the FE mesh of a typical RVE is shown in Fig. 3. It represents the microstructure composed of one single tubule and partially dissolved PTD and ITD. The dimensions of all five RVEs are typical for the tubules and PTD sizes within the range shown in Fig. 2 and listed in Tab.2, but they were arbitrarily designed to examine the individual effect of different PTD fractions (three cases in Group \#1, change in PTD thickness) and dentinal tubule densities (three cases in Group \#2, change in tubule diameter). Note that RVE2 was used as a reference for comparison in each scenario and all the 
other dimensions in each group were designed based on RVE2. In addition, in Group \#2, the PTD thickness was altered to ensure identical PTD fraction in each case.

The dimensions of all five RVEs were firstly adopted in the analytical composite model (Eqs. 1-4) to examine the critical factors that influence the degradation of the effective Young's modulus, with the model size $w=l=5 \mu \mathrm{m}$ in Eq. 3. These RVEs were then used to simulate the incremental dissolution process of the dentine and evaluate its mechanical response, in particular the effective stress redistribution, subjected to a mastication-like load after different stages of dissolution.

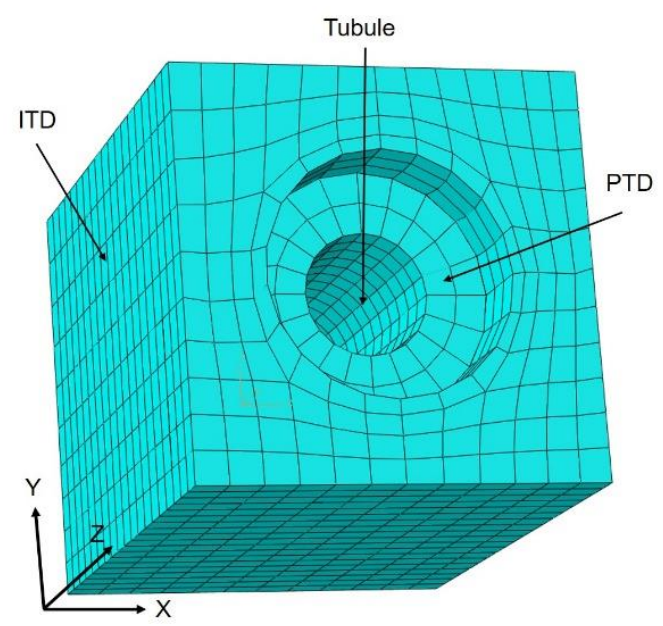

Fig. 3 A typical FE RVE of demineralised dentine that consists of a single tubule surrounded by partially dissolved PTD and ITD.

Table 2 Dimensions of RVEs

\begin{tabular}{|c|c|c|c|c|c|c|}
\hline Description & $\begin{array}{c}\text { RVE } \\
\text { No. }\end{array}$ & $\begin{array}{c}\text { Tubule } \\
\text { diameter } \\
(\boldsymbol{\mu m})\end{array}$ & $\begin{array}{c}\text { PTD } \\
\text { thickness } \\
(\boldsymbol{\mu m})\end{array}$ & $\begin{array}{c}\text { Model } \\
\mathbf{s i z e} \\
(\boldsymbol{\mu m})\end{array}$ & $\begin{array}{c}\text { Initial PTD } \\
\text { fraction } \\
(\boldsymbol{\%})\end{array}$ & $\begin{array}{c}\text { Initial dentinal } \\
\text { tubule density } \\
(\boldsymbol{\%})\end{array}$ \\
\hline \multirow{2}{*}{$\begin{array}{c}\text { PTD fraction } \\
\text { (Group \#1) }\end{array}$} & 1 & 1.5 & 0.25 & 5 & 5.5 & 7 \\
\cline { 2 - 7 } & 2 & 1.5 & 0.75 & 5 & 21.2 & 7 \\
\cline { 2 - 7 } & 3 & 1.5 & 1.25 & 5 & 43.2 & 7 \\
\hline $\begin{array}{c}\text { Dentinal } \\
\text { tubule density } \\
\text { (Group \#2) }\end{array}$ & 4 & 1 & 0.9 & 5 & 21.2 & 3.1 \\
\cline { 2 - 7 } & 5 & 1.5 & 0.75 & 5 & 21.2 & 7 \\
\hline \multirow{2}{*}{} & 2 & 0.65 & 5 & 21.2 & 12.6 \\
\hline
\end{tabular}

\subsection{Computational procedure of dissolution process}

In the FE model described in section 2.2, the original depth of all the RVEs was set identical to be

$7 \mu \mathrm{m}$ based on the dimension of the actual dentine bulk shown in Fig. 1(a). This also covers the full 
dissolution depth shown in Fig. 1(b). The incremental dissolution process of dentine was achieved by adjusting the depth of the top $\left(d_{t}\right)$ and bottom $\left(d_{b}\right)$ layers (initially $d_{t}+d_{b}=7 \mu \mathrm{m}$ ) at each step, where the PTD is absent in the top layer, as described in section 2.1. The bottom surface of the top ITD layer is tied to the top ITD surface of the bottom composite layer. Such constraint is to avoid any interface discontinuity. Following the different dissolution removal depths reported from [22] (see Fig. 1b), for each RVE, the first 20 min dissolution of PTD and ITD was chosen and simulated, as the ITD was found to stabilize after 20 min dissolution (Fig. 1b). Accordingly, 10 steps were made for PTD and ITD respectively to represent the dissolution at every $2 \mathrm{~min}$. The remaining depths of PTD and ITD for each RVE after each step has been summarised in Tab. 1. Note that each 3D RVE is surrounded side-by-side ( $X$-Z and $Y-Z$ planes in Fig. 3 ) and tied by the same RVEs to resemble a periodic boundary condition. However, such condition is not suitable along the $Z$-axis (parallel to the tubule direction, Fig. 3) because of the heterogeneous dissolution between PTD and ITD. Therefore, a fixed boundary condition was used for the bottom surface of each RVE. A constant representative load of $100 \mathrm{MPa}$ was applied to each RVE at the top surface after different steps of acid dissolution. Note that for demineralised RVEs, only ITD on the top layer was effectively subjected to the representative load. To ensure all the RVEs were subjected to an equivalent load, the magnitude of the pressure on the ITD at the top layer was adjusted based on the respective dimensions shown in Tab. 2. The Young's modulus of PTD and ITD was set to be $E_{P T D}=40 \mathrm{GPa}$ and $E_{I T D}=17 \mathrm{GPa}$ respectively from previous nanoindentation results [26]. The higher Young's modulus of PTD is due to its finer-grained and more highly mineralised structure.

After the simulation of all the RVEs, the same simulation procedure was applied on the actual dentine bulk, which was generated as containing 18 dentinal tubules and PTD with different sizes (from previous FIB-SEM reconstruction image, Fig. 1a [7]). This is in conjunction with the RVE simulation results to illustrate the role of PTD from the mechanical perspective. 


\section{Results}

\subsection{Effective Young's Modulus degradation after dissolution}

The analytical composite models described in section 2.1 was used to determine the degradation of effective Young's modulus ( $\left.E_{\text {eff }}\right)$ of all the RVEs (Tab. 2) as well as the dentine bulk sample (Fig. 1a) during the specified acid dissolution process (Tab. 1). The results are shown in Fig. 4, where the solid and dashed lines represent RVEs (the thick black solid line represents the reference RVE2), and the line with symbol represents the dentine bulk sample. $E_{\text {eff }}$ is found to decrease nonlinearly with the vanishing of material, which can be attributed to the faster acid reaction rate of PTD compared with ITD as depicted in Fig. 1(b). The entire dissolution process indicates a decrease of PTD fraction and an increase of dentinal tubule density. The initial $E_{\text {eff }}$ value appears to scale with the fraction of PTD and dentinal tubule density in Tab. 2, with RVE1 giving the lowest value of $E_{\text {eff }}$ while RVE3 giving the highest. However, as the dissolution proceeds, different RVEs exhibit different degrees of reduction in $E_{\text {eff. }}$ When the PTD fraction remains the same (Group \#2), the reduction maintains the same rate. When the PTD fraction varies (Group \#1), $E_{\text {eff }}$ appears to have a higher reduction rate when the initial fraction of PTD is higher. It is also observed that the order of $E_{\text {eff }}$ between different RVEs is altered after some time (e.g. after $12 \mathrm{~min}$ at step 6) of dissolution. For example, RVE4 appears to have the highest $E_{\text {eff }}$ at the end of the considered dissolution process. Finally, the evolution of $E_{\text {eff }}$ of the dentine bulk sample (Fig. 1a) is observed between (and close to) RVE3 and RVE4. Note the calculated PTD fraction and dentinal tubule density of the actual dentine bulk sample are $\sim 32.7 \%$ and $\sim 9.3 \%$ respectively. Compared with the RVE dimensions provided in Tab. 2, the results indicate that PTD fraction is more critical to the effective Young's modulus degradation. 


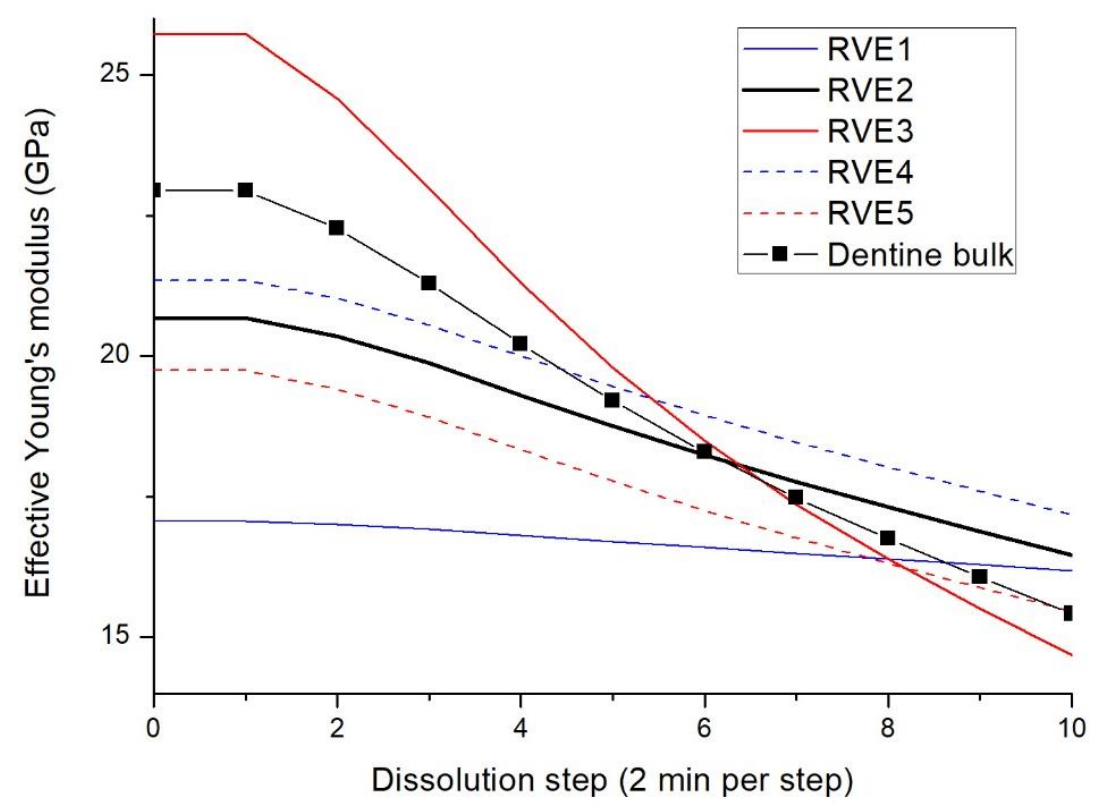

Fig. 4 Degradation of effective Young's modulus of RVEs and the dentine bulk sample as a result of acid dissolution.

\subsection{Effective stress distribution of PTD and ITD after acid dissolution}

\subsubsection{RVE analysis}

Figure 5 presents the effective stress contour plot in the reference RVE2 (Tab. 2) subjected to a representative load after several different acid dissolution stages $(t=0 \mathrm{~min}, 4 \mathrm{~min}, 8 \mathrm{~min}, 12 \mathrm{~min}, 16$ min and $20 \mathrm{~min}$ ). Only half of the RVE (with a cross section parallel to the $Y$ - $Z$ plane in Fig. 3) is presented in order to show the effective stress distribution inside the RVE. A uniform scale in the legend has been used (80-190 MPa) for direct comparison. Before dissolution starts $(t=0 \mathrm{~min})$, stress concentrates predominantly in PTD due to its higher effective Young's modulus than the surrounding ITD. The concentration in PTD appears to become slightly higher towards the bottom. This can be attributed to the constraint by the fixed boundary conditions at the bottom. As the dissolution proceeds, stress is gradually transferred from PTD, in particular close to the top PTD surface, to the top layer without PTD. The maximum stress appears not to change significantly after dissolution. A new stress concentration location can however be observed at the exposed interface between PTD and the surrounding ITD. 


\begin{tabular}{|c|c|c|c|c|c|}
\hline 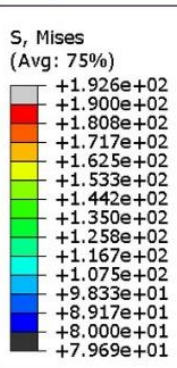 & $\mathrm{t}=0 \mathrm{~min}$ & 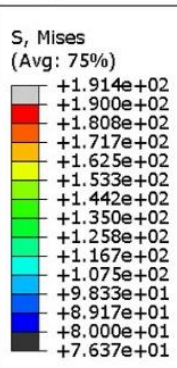 & $\mathrm{t}=4 \mathrm{~min}$ & 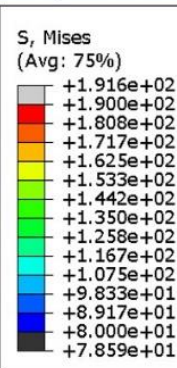 & $\mathrm{t}=8 \mathrm{~min}$ \\
\hline 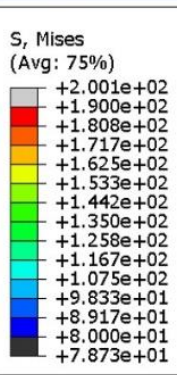 & $\mathrm{t}=12 \min$ & 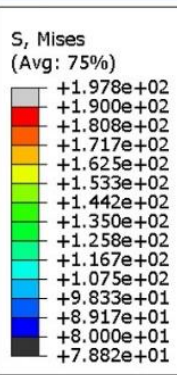 & $\mathrm{t}=16 \mathrm{~min}$ & 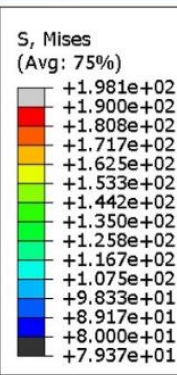 & $20 \mathrm{mir}$ \\
\hline
\end{tabular}

Fig. 5 Distribution of effective stress in RVE2 subjected to a constant load after selected steps of acid dissolution: (a) $t=0 \mathrm{~min}$; (b) $t=4 \mathrm{~min}$; (c) $t=8 \mathrm{~min}$; (d) $t=12 \mathrm{~min}$; (e) $t=16 \mathrm{~min}$; (f) $t=20 \mathrm{~min}$. A uniform legend (80$190 \mathrm{MPa}$ ) is used for comparison. The cross section is parallel to the $Y$ - $Z$ plane in the centre of the RVE.

Figure 6 compares the effective stress contour plot in all the RVEs between the two different groups after the last stage of acid dissolution $(t=20 \mathrm{~min})$. The result in the reference RVE2 is shown twice (Fig. 6b \& e) for comparison with other RVEs. The same uniform scale in the legend (80-190 MPa) has been used. Overall, the greater variation in the effective stress distribution is observed in Group \#1 (variation in PTD fraction) compared with Group \#2 (variation in dentinal tubule density). Similar to Fig. 5, stresses tend to concentrate at the exposed interface between PTD and the surrounding ITD, which is where the maximum effective stress exists. The area for the concentration, however, differs in different RVEs. An obvious trend can be seen such that the stress in the bottom layer (resemble the original RVE but with a reduced depth) gradually transfers to the top layer as the initial PTD fraction increases in Group \#1 (RVE1->RVE2->RVE3). Such trend is less obvious when the dentinal tubule density varies while the PTD fraction remains the same in Group \#2. The worst scenario occurs in RVE3 originally having the highest PTD fraction, where the stress for the majority of the top layer exceeds that of the bottom layer. 


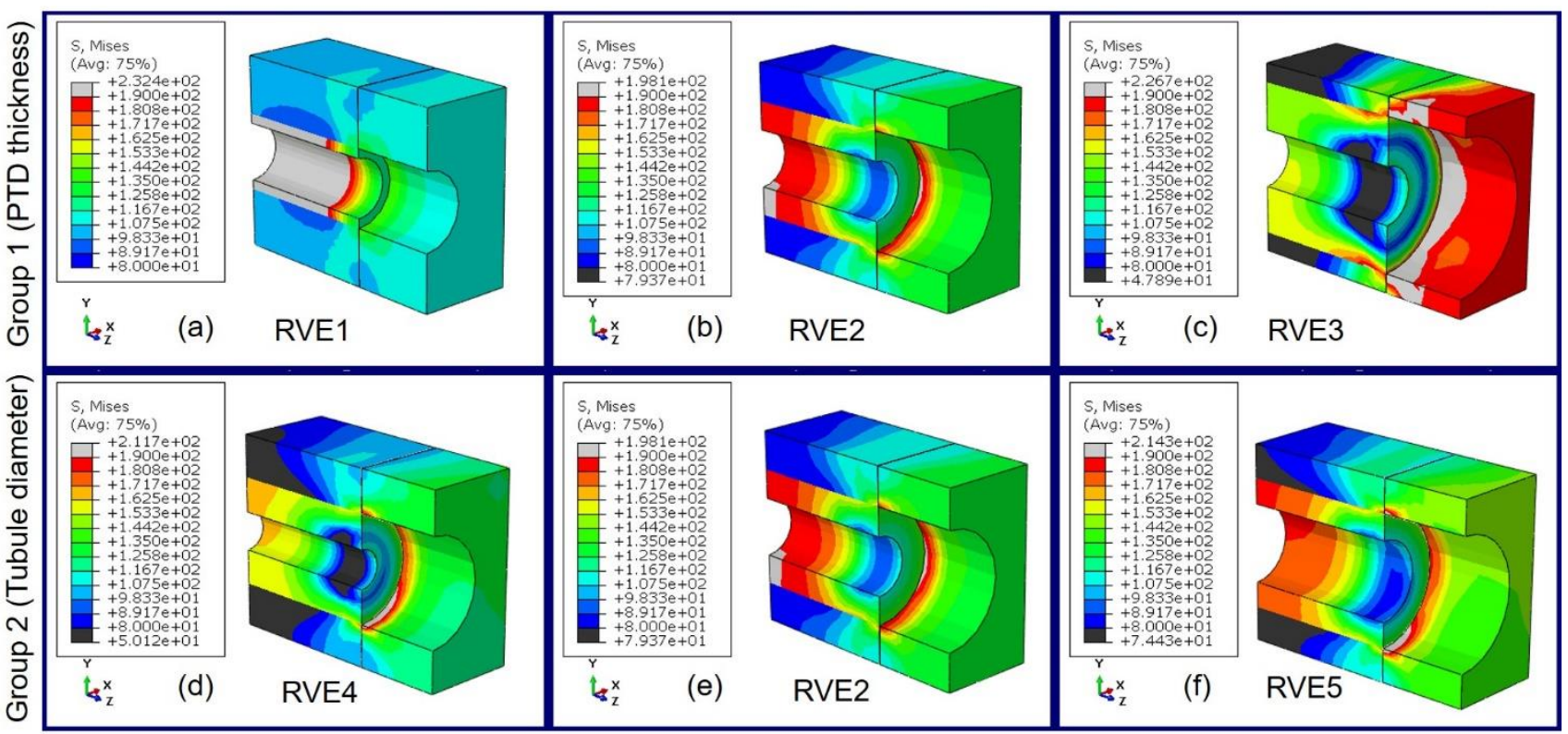

Fig. 6 Distribution of effective stress in all the RVEs (arranged in two different groups, Group \#1 for PTD fraction, Group \#2 for dentinal tubule density) subjected to an equivalent representative load along the tubule direction after the last stage of acid dissolution $(t=20 \mathrm{~min})$. A uniform legend (80-190 MPa) is used. The cross section is parallel to the $Y-Z$ plane in the centre of the RVE.

\subsubsection{Bulk analysis}

Figure 7 presents the effective stress contour plot in the dentine bulk sample (depicted in Figs. 1 and 2) at several selected stages of acid dissolution ( $t=0 \mathrm{~min}, t=10 \mathrm{~min}, t=20 \mathrm{~min}$ ). Figs. 7(a)-(c) show the effective stress distribution viewed from the top surface perpendicular to the loading direction, whereas Figs. 7(d)-(f) provide the effective stress distribution inside the bulk at a cross section parallel to the $Y-Z$ plane in Fig. 2. The same uniform scale in the legend (80-190 MPa) as Figs. 5 and 6 has been used. Similar trend with that in Fig. 5 can be observed such that the load is gradually transferred to the top layer without PTD as the dissolution proceeds. As the actual dentine bulk sample contains different tubule diameters and PTD thicknesses compared with the proposed RVEs, some subtle differences can be found. For example, before dissolution, not all the PTDs experience the same stress. The ITD in the centre regions surrounded by dense PTDs close to the edge of the bulk seem to be well protected with relatively low stress compared with those near the edge surrounded by loose PTDs, which seems to be independent of the dissolution stage. Apart from the stress concentrations occurring 
at the PTD-ITD interfaces in the composite after dissolution, additional concentrations can be seen at the top layer ITD, connecting the "big holes" like a "chain" shape (e.g. see the enlarged tubules near the right hand side edge in Fig. $7 b$ \&c).

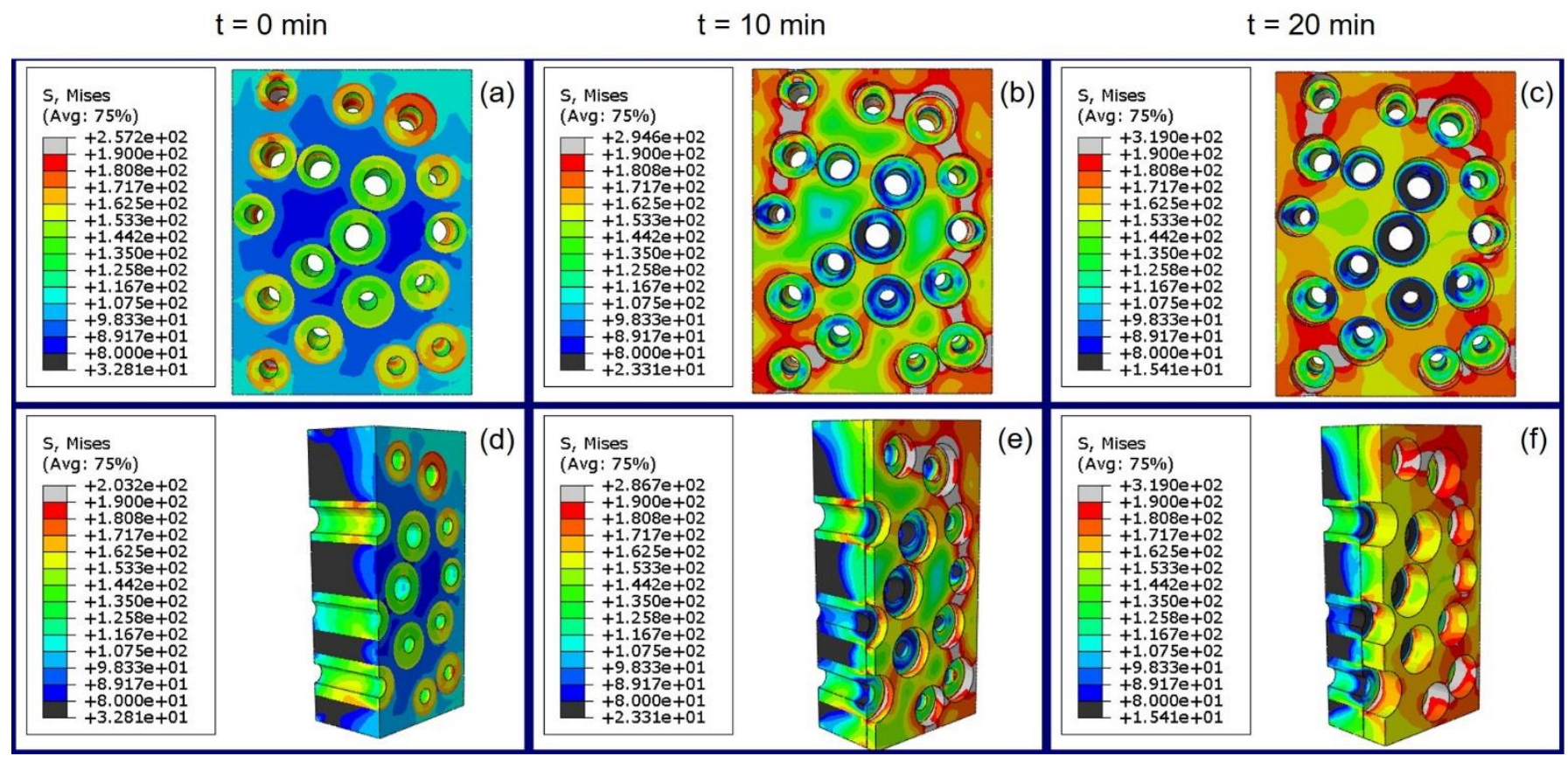

Fig. 7 Distribution of effective stress in the actual dentine bulk sample subjected to an equivalent representative load at several selected stages of dissolution $(t=0 \mathrm{~min}, t=10 \mathrm{~min}, t=20 \mathrm{~min})$. A uniform legend (80-190 MPa) is used. The cross section is parallel to the $Y$ - $Z$ plane, cutting three tubules and adjacent PTD and ITD.

\section{Discussion}

The calculated initial value of effective Young's modulus of the intact dentine bulk sample has been found to match the numerical and analytical results reported in the work by Grzebieluch et al [27]. The comparison of the degradation of the effective Young's modulus shown in Fig. 4 exhibits a correlation with the fraction of PTD and dentinal tubule density at the initial condition. Hence it is capable of using simple analytical model to resemble the complex dentine bulk provided the same fraction of PTD and dentinal tubule density. If the dissolution continues beyond 20 min shown in Fig. 1(b), the effective Young's modulus of all the RVEs as well as the dentine bulk would asymptote to the respective value for the top layer with pure ITD and "enlarged" tubules. Some RVEs initially with high effective Young's modulus (e.g. RVE3) gradually becomes inferior to some RVEs initially with 
low effective Young's modulus (e.g. RVE4) at later stages of dissolution. This is because after dissolution, RVEs with more fraction of PTD at the beginning would decompose to a very soft top layer and a hard bottom layer with relatively sharper contrast in the effective Young's modulus. The general trend of the degradation for the dentine bulk sample has been found to agree reasonably well with the experimental findings using nanoindentation in carious human dentine samples [28], despite that the experiment has reported a large range of lowest effective Young's modulus values at the outer lesion cavity floor of carious dentine $(0.015$ to $14.55 \mathrm{GPa})$ which is partially beyond the value the current approach can predict. This has been explained such that some localised outer regions might be comprised mostly of denatured collagen [28-30] rather than completely be etched away. In this scenario, the top layer would instead become a composite of collagen and enlarged tubules. Such complexity could be captured by modelling the structure with a gradient in properties, which is beyond the scope of this study. A clear novelty in this study is the systematic investigation of the effect of variation in PTD fraction and dentinal tubule density on the mechanical property degradation during acid dissolution. In practice, a degradation of effective Young's modulus indicates that the demineralised dentine bulk would suffer from more deformation given the same representative load compared with the intact dentine. As the PTD fraction is found to be more critical than the dentinal tubule density, a high fraction of PTD would increase the severity of the effect of acid dissolution, which has been clearly reflected from the effective stress distribution in the RVEs shown in Figs. 5-6 (e.g. RVE3 in Fig. 6c).

The numerical simulation has also highlighted the important role of PTD concerning the effective stress redistribution, demonstrating its importance to the structural integrity of the entire dentine. The effective stress distribution in PTD and ITD in the RVEs and dentine bulk (Figs. 5-7) reveals that in most cases the PTD takes more load than ITD, functioning as a protection constituent to the surrounding ITD and also the entire dentine bulk. However, owing to the lack of numerical study on the effect of acid dissolution, the results in this study provide new findings and qualitative insight to 
the understanding of the role of PTD. Both the stress concentration at the interfaces between PTD and ITD and the load transfer to ITD from PTD after extensive acid dissolution of PTD indicate the weakening of its mechanical protection effect for ITD and the reinforcement to the entire dentine. The occurrence of stress concentration at the interfaces can be attributed to the change in the constraint circumstances, while the load transfer can be explained by the variation in the effective Young's modulus between the two layers. Before the acid dissolution, the ITD is well protected by the PTD so the principal stress in ITD is mainly along the direction of the applied load ( $Z$-axis). After the acid dissolution, while such stress increases in the top layer, the exposed ITD surface at the top layer also becomes free from the in-plane ( $X-Y$ plane) deformation perpendicular to the applied load. Close to the PTD surface at the bottom layer, the ITD is still partly subjected to the constraint from the PTD underneath. As a consequence, a net effect is the occurrence of an enhanced in-plane stress state at the PTD-ITD interfaces, which subsequently leads to the concentration of the effective stress. The higher the PTD fraction is before the acid dissolution, the larger area the concentration would be at the interface after extensive acid dissolution (see Fig. 6). Such relaxation of the constraint circumstances can also explain the "chain shape" concentration (see Fig. 7) in the dentine bulk when a number of tubules and PTDs are irregularly distributed. Extremely high stress concentration appears at the top layer ITD where the expanded holes are very close to each other. This can be attributed to the localised volume with very high fraction of PTD before dissolution, which makes that part of dentine susceptible and vulnerable to dissolution. Further, note that change of the predominant stress concentration location from PTD to ITD and interfaces may increase the risk of crack nucleation in ITD, which is a potential precursor of catastrophic failure in the entire dentine. Therefore, the presence of intact PTD is essential to the structural integrity of the whole dentine.

The proposed numerical incremental dissolution process has been shown to be capable of capturing the mechanical response of dentine subjected to a representative load after different stages of acid dissolution. A broader application of the proposed approach in this study can be the mechanical 
performance evaluation of human dental caries before and after surgical treatments. Complex dynamic chemical processes such as de- and remineralisation (dissolution and formation of different crystallite phases) can occur in human dental caries and some of its treatments [31,32]. These chemical processes may result in gradient mechanical properties in the PTD and ITD other than uniform.

\section{Conclusion}

An analytical and a 3D numerical investigation of the mechanical performance and degradation of the mechanical property of demineralised human dentine is presented. The fraction of PTD is found to be the most critical factor for the degradation of effective Young's modulus, effective stress redistribution and concentration of the representative dentine bulk as a result of acid dissolution. The results highlight the importance of PTD concerning the structural integrity of entire dentine. On one hand, it strengthens the overall dentine system while prevents the occurrence of stress concentration in ITD. On the other hand, its more susceptibility to acid exposure helps protect ITD from severe chemical dissolution and crack initiation. These obtained insights are crucial for advancing the understanding and modelling of a variety of natural and therapeutic effects from the mechanical perspective in dentine tissue development, which would benefit further bioinspired materials manufacturing and design for restoring the materials and functions of human dental tissues. Further refinement and validation of the proposed model will be sought in the future considering in situ experiment such as digital volume correlation (DVC) analysis for 3D strain evaluation across entire material volumes.

\section{Author contributions}

Jianan $\mathrm{Hu}$ and Tan Sui conceived the study. Jianan Hu carried out the analytical study and numerical simulation. Tan Sui guided the RVE selection and further results analysis. Jianan Hu and Tan Sui wrote the manuscript. Both authors commented on the manuscript. 


\section{Conflict of interest statement}

The authors have no conflicts of interest to disclose.

\section{References}

[1] R. Schilke, J.A. Lisson, O. Bauss, W. Geurtsen, Comparison of the number and diameter of dentinal tubules in human and bovine dentine by scanning electron microscopic investigation, Arch Oral Biol 45(5) (2000) 355-61.

[2] M.B. Lopes, M.A. Sinhoreti, A. Gonini Junior, S. Consani, J.F. McCabe, Comparative study of tubular diameter and quantity for human and bovine dentin at different depths, Braz Dent J 20(4) (2009) 279-83.

[3] A.R. Ten Cate, A.C. Dale, Oral histology : development, structure, and function, Mosby, St. Louis, 1980.

[4] L.M. Petrovic, D.T. Spasic, T.M. Atanackovic, On a mathematical model of a human root dentin, Dent Mater 21(2) (2005) 125-8.

[5] D.H. Pashley, B. Ciucchi, H. Sano, R.M. Carvalho, C.M. Russell, Bond strength versus dentine structure: a modelling approach, Arch Oral Biol 40(12) (1995) 1109-18.

[6] R. Wang, L. Niu, Q. Li, Q. Liu, H. Zuo, The peritubular reinforcement effect of porous dentine microstructure, PLoS One 12(8) (2017) e0183982.

[7] T. Sui, J. Dluhos, T. Li, K. Zeng, A. Cernescu, G. Landini, A.M. Korsunsky, Structure-Function Correlative Microscopy of Peritubular and Intertubular Dentine, Materials (Basel) 11(9) (2018).

[8] S. Weiner, A. Veis, E. Beniash, T. Arad, J.W. Dillon, B. Sabsay, F. Siddiqui, Peritubular dentin formation: crystal organization and the macromolecular constituents in human teeth, J Struct Biol 126(1) (1999) 27-41.

[9] S.R. Stock, A.C. Deymier-Black, A. Veis, A. Telser, E. Lux, Z. Cai, Bovine and equine peritubular and intertubular dentin, Acta Biomater 10(9) (2014) 3969-77.

[10] S. Habelitz, B.J. Rodriguez, S.J. Marshall, G.W. Marshall, S.V. Kalinin, A. Gruverman, Peritubular dentin lacks piezoelectricity, J Dent Res 86(9) (2007) 908-11.

[11] L.E. Bertassoni, K. Stankoska, M.V. Swain, Insights into the structure and composition of the peritubular dentin organic matrix and the lamina limitans, Micron 43(2-3) (2012) 229-36.

[12] M. Sezen, S. Sadighikia, 3D electron microscopy investigations of human dentin at the micro/nano-scale using focused ion beam based nanostructuring, RSC Advances 5 (2015) 7196-7199.

[13] L.G. Watanabe, G.W. Marshall, Jr., S.J. Marshall, Dentin shear strength: effects of tubule orientation and intratooth location, Dent Mater 12(2) (1996) 109-15.

[14] V. Lertchirakarn, J.E. Palamara, H.H. Messer, Anisotropy of tensile strength of root dentin, J Dent Res 80(2) (2001) 453-6.

[15] M. Balooch, I.C. Wu-Magidi, A. Balazs, A.S. Lundkvist, S.J. Marshall, G.W. Marshall, W.J. Siekhaus, J.H. Kinney, Viscoelastic properties of demineralized human dentin measured in water with atomic force microscope (AFM)-based indentation, J Biomed Mater Res 40(4) (1998) 539-44.

[16] T. Sui, M.A. Sandholzer, N. Baimpas, I.P. Dolbnya, A. Walmsley, P.J. Lumley, G. Landini, A.M. Korsunsky, Multiscale modelling and diffraction-based characterization of elastic behaviour of human dentine, Acta Biomater 9(8) (2013) 7937-47.

[17] S. Seyedkavoosi, I. Sevostianov, Multiscale micromechanical modeling of the elastic properties of dentin, J Mech Behav Biomed Mater 100 (2019) 103397.

[18] J.H. Kinney, M. Balooch, G.W. Marshall, S.J. Marshall, A micromechanics model of the elastic properties of human dentine, Arch Oral Biol 44(10) (1999) 813-22.

[19] J.H. Kinney, M. Balooch, S.J. Marshall, G.W. Marshall, Jr., T.P. Weihs, Hardness and Young's modulus of human peritubular and intertubular dentine, Arch Oral Biol 41(1) (1996) 9-13.

[20] B. An, Analysis of crack interacting with the composite microstructure of dentin, European Journal of Mechanics A/Solids 66 (2017) 287-295. 
[21] T. Sui, A.J. Lunt, N. Baimpas, M.A. Sandholzer, J. Hu, I.P. Dolbnya, G. Landini, A.M. Korsunsky, Hierarchical modelling of in situ elastic deformation of human enamel based on photoelastic and diffraction analysis of stresses and strains, Acta Biomater 10(1) (2014) 343-54.

[22] J.H. Kinney, M. Balooch, D.L. Haupt, Jr., S.J. Marshall, G.W. Marshall, Jr., Mineral distribution and dimensional changes in human dentin during demineralization, J Dent Res 74(5) (1995) 1179-84.

[23] G.W. Marshall, Jr., M. Balooch, R.J. Tench, J.H. Kinney, S.J. Marshall, Atomic force microscopy of acid effects on dentin, Dent Mater 9(4) (1993) 265-8.

[24] J.L. Nino-Barrera, M.L. Gutierrez, D.A. Garzon-Alvarado, A theoretical model of dentinogenesis: dentin and dentinal tubule formation, Comput Methods Programs Biomed 112(1) (2013) 219-27.

[25] Q.H. Qin, M.V. Swain, A micro-mechanics model of dentin mechanical properties, Biomaterials 25(20) (2004) 5081-90.

[26] D. Ziskind, M. Hasday, S.R. Cohen, H.D. Wagner, Young's modulus of peritubular and intertubular human dentin by nano-indentation tests, J Struct Biol 174(1) (2011) 23-30.

[27] W. Grzebieluch, R. Bedzinski, T. Czaplinski, U. Kaczmarek, The mechanical properties of human dentin for 3-D finite element modeling: Numerical and analytical evaluation, Adv Clin Exp Med 26(4) (2017) 645653.

[28] L. Angker, M.V. Swain, N. Kilpatrick, Characterising the micro-mechanical behaviour of the carious dentine of primary teeth using nano-indentation, J Biomech 38(7) (2005) 1535-42.

[29] T. Fusayama, K. Okuse, H. Hosoda, Relationship between hardness, discoloration, and microbial invasion in carious dentin, J Dent Res 45(4) (1966) 1033-46.

[30] K. Ogawa, Y. Yamashita, T. Ichijo, T. Fusayama, The ultrastructure and hardness of the transparent layer of human carious dentin, J Dent Res 62(1) (1983) 7-10.

[31] L.E. Bertassoni, S. Habelitz, S.J. Marshall, G.W. Marshall, Mechanical recovery of dentin following remineralization in vitro--an indentation study, J Biomech 44(1) (2011) 176-81.

[32] D.J. Epasinghe, C.K. Yiu, M.F. Burrow, J.K. Tsoi, F.R. Tay, Effect of flavonoids on the mechanical properties of demineralised dentine, J Dent 42(9) (2014) 1178-84. 